\title{
Review Article \\ Whole-Body Vibration Exercise for Knee Osteoarthritis: A Systematic Review and Meta-Analysis
}

\author{
Xin Li, ${ }^{1}$ Xue-Qiang Wang, ${ }^{2,3}$ Bing-Lin Chen, ${ }^{2}$ Ling-Yan Huang, ${ }^{1}$ and Yu Liu ${ }^{1}$ \\ ${ }^{1}$ Key Laboratory of Exercise and Health Sciences of the Ministry of Education, Shanghai University of Sport, Heng Ren Road, \\ No. 200, Yang Pu District, Shanghai 200438, China \\ ${ }^{2}$ School of Kinesiology, Shanghai University of Sport, Qing Yuan Huan Road, No. 650, Yang Pu District, Shanghai 200438, China \\ ${ }^{3}$ Department of Sport Rehabilitation, Shanghai University of Sport, Chang Hai Road, No. 399, Yang Pu District, \\ Shanghai 200438, China \\ Correspondence should be addressed to Yu Liu; yuliu@sus.edu.cn
}

Received 9 April 2015; Revised 22 June 2015; Accepted 2 July 2015

Academic Editor: Martin Offenbaecher

Copyright (C) 2015 Xin Li et al. This is an open access article distributed under the Creative Commons Attribution License, which permits unrestricted use, distribution, and reproduction in any medium, provided the original work is properly cited.

Objectives. To assess the effects of WBV exercise on patients with KOA. Methods. Eight databases including Pubmed, EMBASE, Cochrane Library, CINAHL, Web of Science, the Physiotherapy Evidence Database, CNKI, and Wanfang were searched up to November 2014. Randomized controlled trials (RCTs) of WBV for KOA were eligible. The outcomes were pain intensity, functional performances, self-reported status, adverse events, and muscle strength. A meta-analysis was conducted. Results. Five trials with 168 participants provided data for the meta-analysis. No significant difference was shown in pain intensity and self-reported status between WBV and other forms of exercise. Improvement in functional performance (evaluated by BBS; WMD, 2.96; $95 \%$ CI, 1.29 to 4.62; $P=0.0005$ ) was greater in WBV group, but the other parameters of functional performance (including 6MWT and TGUG) revealed no statistically significant difference. Adverse events were only reported in one trial and no significant difference was discovered in muscle strength. The overall quality of evidence was very low. Conclusion. Currently there is only limited evidence that suggested that WBV is effective in the treatment of KOA. Large, well-designed RCTs with better designs are needed.

\section{Introduction}

Osteoarthritis (OA) is a chronic, ingravescent, and degenerative osteoarticular disease with a multifactorial etiology [1]. OA is characterized by arthralgia, stiffness, and limitations in articular function [1-3]. Researchers at the World Health Organization forecast that OA will become the fourth primary cause of disability by the year 2020 [4]. Felson [5] reported that approximately one-third of adults worldwide showed radiological signs of OA. Andrianakos et al. [6] reported significant hand, knee, or hip OA in $8.9 \%$ of the adult population. Thus far, the substantial morbidity of knee OA (KOA) has affected approximately $28 \%$ of the population aged 45 years and older and $37 \%$ of adults older than 65 years of age in the United States [7]. KOA is now one of the leading joint diseases that causes pain, stiffness, loss of physical function, and other adverse effects among adults [8]. The burdens on health care resources and on the economy caused by KOA are substantial $[9,10]$. Various forms of exercise, including walking, balance training, resistance training, hydrotherapy, and Tai Chi training, have been investigated as potential methods of KOA management [11].

Whole-body vibration (WBV) exercise is a feasible and curative strength-exercise technique that has received considerable attention in recent years [12]. The vibrations are generated by a vibrating plate and are transmitted from surfaces in contact with the human body to stimulate muscles and tendons. WBV provides a time benefit compared with other traditional resistance exercise programs [13].

During the past decade, some studies have found that WBV may enhance muscle strength and power and may have the potential to be a useful adjuvant in physiotherapy and health care [13]. A large number of studies have assessed the efficiency of WBV on muscle strength and power enhancement [14-20]. Studies have reported that WBV is helpful in improving neuromuscular performance and in providing 
a safe exercise program for elderly people $[19,21,22]$. WBV has also been found to enhance the multijoint strength performance of the lower limbs during a countermovement jump [23]. A recent meta-analysis also reported that WBV may be useful in increasing the relative basic mobility and balance ability of older adults, particularly among the frail ones [24]. The UK National Institute for Health and Clinic Excellence recommended physical activity and exercise as the core treatment for KOA in enhancing muscle strength and lower extremity multijoint strength [25]. Furthermore, basic balance ability and mobility are highly correlated with the improvement of knee-specific function [26]. Thus, WBV may be beneficial for patients with knee OA.

Ronikeile and Costa [27] reported the benefits of WBV for people with $\mathrm{OA}$ by systematically reviewing several studies. Several trials have reported that WBV can alleviate pain, improve balance control, and improve gait pattern and other parameters $[28,29]$. However, some trials failed to find significant improvements in pain intensity or other parameters among patients with KOA [30, 31]. Thus, the benefits of WBV on KOA management remain unclear. Thus far, no meta-analysis has evaluated evidence on pain intensity, functional performance, self-reported status, and adverse events. This study aims to evaluate the effect of WBV exercise by comparing WBV with other exercises via meta-analyses for patients with KOA.

\section{Methods}

2.1. Literature Search. We authenticated relevant articles by conducting electronic searches on the following databases from the earliest available date to November 2014: PubMed, EMBASE, Cochrane Library, Cumulative Index Nursing and Allied Health Literature, Web of Science, the Physiotherapy Evidence Database, China National Knowledge Infrastructure, and the Wanfang Database. We used terms such as "knee osteoarthritis," "whole-body vibration," and "random" in the databases above (File S1 shows the concrete details of the search in Supplementary Material available online at http://dx.doi.org/10.1155/2015/758147). No year, language, or status restrictions were applied to enhance the probability of obtaining interrelated publications that are related to the effect of WBV and KOA.

To identify gray literature, domain experts were consulted and we investigated data that were not represented in the aforementioned databases. Furthermore, the International Controlled Trials Registry Platform was retrieved to search for relevant conference and other literature that might have contained additional data by using "whole-body vibration" and "knee osteoarthritis" as keywords. However, we did not obtain any additional published papers. We also manually screened the reference lists of consilient publications to obtain articles.

\subsection{Inclusion Criteria}

2.2.1. Types of Studies. Randomized controlled trials (RCTs) that compared exercises with or without WBV or compared only WBV exercise with no intervention or placebo group were acceptable. There were no restrictions on year, language, status, or publication date.

2.2.2. Types of Participants. The subjects in the trials included patients who met the diagnostic criteria of definite KOA for at least three months. Subjects exposed to analogous treatments before the study were excluded unless an adequate washout period was described.

2.2.3. Types of Interventions. Trials that compared WBV treatment with no exercise, sham-WBV treatment, or other exercise interventions for KOA were included. Studies in which combinations of interventions were performed were acceptable as long as these combinations were controlled for in the structure of the trial.

2.2.4. Types of Outcome Measures. The results were summarized and analyzed according to four primary outcome classifications: (1) pain intensity, (2) functional performance, for example, Berg balance scale (BBS), 6 min walk test (6MWT), and timed get up and go test (TGUG), (3) self-reported status (using WOMAC scale), and (4) adverse events. The secondary outcome was muscle strength (e.g., extensor peak isokinetic torque, extensor peak isometric torque, and flexor peak isokinetic torque).

2.3. Selection of Studies. Two reviewers (Li, X. and Liu, Y.) independently sorted through the articles for potentially relevant titles. Abstracts of all identified records were each screened by two reviewers, and articles were retrieved in full whenever necessary. Any discord was settled by discussing or consulting with another reviewer (Wang, X. Q.) if necessary.

2.4. Quality Assessment. To identify the methodological quality of all included trials, the risk of bias tool of the Cochrane Collaboration was applied by two independent authors (Li, X. and Liu, Y.). The evaluated issues included random sequence generation, allocation concealment, blinding of participants and personnel, blinding of outcome assessments, incomplete outcome data, and selective reporting [32]. As far as each domain was concerned, the methods described in each study were examined and potential bias was evaluated in accordance with three grades: low risk, high risk, and unclear risk $[27,33]$. Disagreements were resolved after consulting a third independent author (Wang, X. Q.).

2.5. Data Extraction. Two authors (Li, X. and Liu, Y.) independently extracted and crosschecked the data acquired from every included trial. The study design (random sequence generation, allocation concealment, blinding of participants and personnel, blinding of outcome assessments, incomplete outcome data, and selective reporting) was then recorded. Any discord was settled by conference to reach unanimity. Authors were contacted directly to acquire the original studies and data when necessary.

2.6. Statistical Analysis. The effects of WBV on outcomes of interest were analyzed. Meta-analysis was performed if 
TABLE 1

\begin{tabular}{lccc}
\hline & Group 1 & Group 2 & Combined groups \\
\hline Sample size & $N_{1}$ & $N_{2}$ & $N_{1}+N_{2}$ \\
Mean & $M_{1}$ & $M_{2}$ & $\left(N_{1} M_{1}+N_{2} M_{2}\right) /\left(N_{1}+N_{2}\right)$ \\
$\mathrm{SD}$ & $\mathrm{SD}_{1}$ & $\mathrm{SD}_{2}$ & $\sqrt{\left(\left(N_{1}-1\right) \mathrm{SD}_{1}^{2}+\left(N_{2}-1\right) \mathrm{SD}_{2}^{2}+\left(N_{1} N_{2} /\left(N_{1}+N_{2}\right)\right)\left(M_{1}^{2}+M_{2}^{2}-2 M_{1} M_{2}\right)\right) /\left(N_{1}+N_{2}-1\right)}$ \\
\hline
\end{tabular}

two or more studies evaluated the same outcome. For each outcome of interest in the selected study, the weighted mean difference (WMD) and 95\% confidence interval (CI) were computed. The data was analyzed by Review Manager statistical software (RevMan 5.2, The Cochrane Centre, The Cochrane Collaboration, Denmark) by using a randomeffects model. The proportion of variance in the pooled estimates caused by heterogeneity was evaluated by the following parameters: $I^{2}$ index: $<25 \%$, low heterogeneity; $<75 \%$, moderate heterogeneity; and $\geq 75 \%$, high heterogeneity [34]. No funnel plots analysis would be performed, if the number of trials pooled in the comparison included in this literature was quite small (maximum of three trials).

The control conditions included both no treatment and sham-WBV treatment and other forms of exercise, including home-based exercise, squat exercise, and balance board exercise. If one trial contained two or more matched groups that performed various exercise programs (e.g., other forms of exercises including home-based exercise, squat exercise, and balance board exercise), the data from the control groups were combined by using the equation in Table 1 .

Analyses were possible only for pain intensity, functional performance, self-reported status, and muscle strength because all data on adverse events originated from one article only. All the variables were continuous in the analysis. Therefore, a random-effects model was applied. Two-sided statistical tests were used. $P<0.05$ or $95 \%$ CI was deemed a statistically significant difference [35].

2.7. Data Synthesis. Grading of Recommendations Assessment, Development and Evaluation (GRADE) system was used to rate the quality of evidence for each outcome across studies. The system allows for classifying the quality of evidence into high quality, moderate quality, low quality, or very low quality, depending on study design, study limitations (risk of bias), inconsistency, indirectness of study results, imprecision, and publication bias [36]. The judging criteria are listed in Appendix S1.

\section{Results}

3.1. Study Selection. Our first search initially yielded 13220 records. After reviewing the information in the titles and abstracts, 750 papers were read in detail. Finally, 5 RCTs [28-31, 37] (168 participants) with valid outcome data met the inclusion criteria and were included in this analysis. The process of identifying these studies from initial publication searches to final inclusion is illustrated in Figure 1.

3.2. Description of Included Studies. Five RCTs with the purpose of examining the efficacy of WBV on KOA $[28-31,37]$ were included in this meta-analysis. The trials were published in English from 2009 to 2014. The time of intervention ranged from 8 weeks to 12 weeks (median: 9.6 weeks). Among the trials, two trials were from Brazil $[29,31]$, whereas the three other studies were from Korea [30], Denmark [37], and Finland [28]. Trials recruited participants with KOA mostly from local hospitals and clinics. Across the included studies, three different devices were used for the whole-body vibration exercise. Three studies used vertical vibration $[28$, 29,31 ], one study used side-alternating vibration [37], and one study used multidirectional vibration [30]. Across all five studies the frequency of the vibration used varied from 12 to $40 \mathrm{~Hz}$. Study by study, the reported frequencies of vibration were 12 to $14 \mathrm{~Hz}$ [30], 25 to $30 \mathrm{~Hz}$ [37], 35 to $40 \mathrm{~Hz}$ [29, 31], and $30 \mathrm{~Hz}$ [28]. Four studies documented the amplitude of the vibration, reporting amplitudes of $4 \mathrm{~mm}[29,31], 2.5-$ $5 \mathrm{~mm}$ [30], and $2.5 \mathrm{~mm}$ [28]. One study did not report the amplitude of the vibration [37]. Table 2 elaborates the details of the included studies, such as the subjects' characteristics, sample size, intervention, duration of trial period, outcomes, and time points. All the durations of intervention were less than 6 months, and we did not categorize outcomes into different lengths of intervention period.

3.2.1. Participants. We extracted data from 168 participants (number of subjects in the study was from 21 to 52), among whom 64 were exposed to WBV only and 34 received WBV combined with other therapies (11 combined with homebased exercise and 23 combined with squat exercise). The average age of the participants ranged from 58.7 years to 75 years. Subjects were all from hospitals/clinics and were included according to various inclusion criteria.

3.2.2. $W B V$. The intervention was foremost WBV based, and two $[29,31]$ of the five trials involved the squat exercises along with the WBV intervention. Exercise duration ranged from 8 weeks to 12 weeks with a mean length of approximately 9.6 weeks. The intervention was performed two or three times a week. Four trials were conducted in the hospital and one was conducted in the clinic. In three of the included studies [29, 31,37 ], the vibration time (20-70 s) increased systematically with the number of repetitions (6-9 repetitions). One study was performed when the participants conducted a series of acceleration exercise programs [28]. In another study, participants spent 20 min per session doing WBV [30].

3.2.3. Control Conditions. Two studies used home-based exercises $[28,30]$ and one study used two independent control interventions, which included squat exercise only and no treatment [29]. One trial used squat exercises as control [31] and the other one used no exercise [37]. 


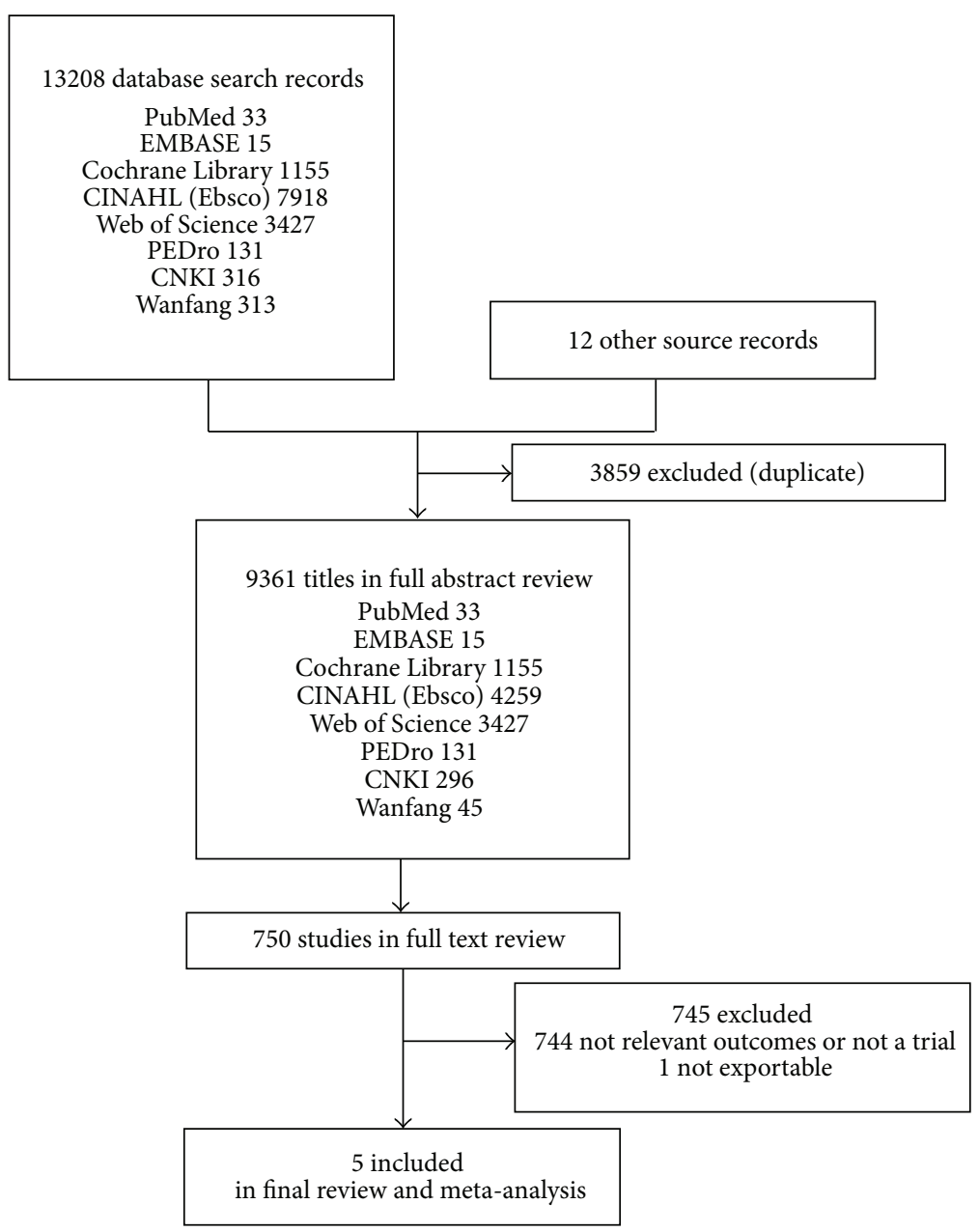

FIGURE 1: Review flow diagram.

3.2.4. Outcomes. Primary outcome measures were pain intensity [28, 30], self-reported status [29, 31, 37], and functional performance $[28,29,31]$ (including BBS [29, 31], 6MWT [29, 31], and TGUG [28, 31]). However, none of the included trials reported adverse events. The secondary outcome measure was muscle strength $[28,30,31,37]$.

3.3. Methodological Quality of Included Trials. Following recommendations, the risk of bias was assessed in every included trial and we found that the amount of methodological details varied. For example, several quality indexes were insufficiently described in the articles (Table 3). All of the five trials [28-31, 37] described the randomization method that was being used, but three [28, 30, 31] reported no information on treatment allocation concealment. The blinding of assessors was used in four studies [28, 29, 31, 37]. None of the five included trials [28-31, 37] met the requirements for the blinding of participants. However, in view of the characteristics in therapeutic interventions and direct participant-personnel involvement, the blinding of participants was deemed unfeasible, although the absence of blinding must be viewed as a potential source of bias. By contrast, a low risk of bias about incomplete outcome data was only reported in one trial. It was unclear if all five trials were free of selective outcome reporting. Since the assessment of publication bias was only feasible for those comparisons that included a minimum of three studies, we failed to assess other bias. Overall, the methodological quality of the five included trials was judged to be highly risky. What is more, given that the number of trials pooled in the comparison included in this literature was quite small (maximum of three trials), no funnel plots analysis was performed. The quality of evidence was downgraded by very serious study limitations and imprecision. Therefore, there was very low quality evidence overall.

\subsection{Effectiveness}

3.4.1. Pain Intensity. Two trials randomly assigned patients to undergo WBV combined with/without home-based exercise or home-based exercise only $[28,30]$. Of these two studies, one trial evaluated pain intensity by using a $100 \mathrm{~mm}$ visual analog scale (VAS) [28] and the other by using a 0-point to 10-point numerical rating scale (NRS) [30]. Moderate to high correlations exist between these two different measures [38]. 


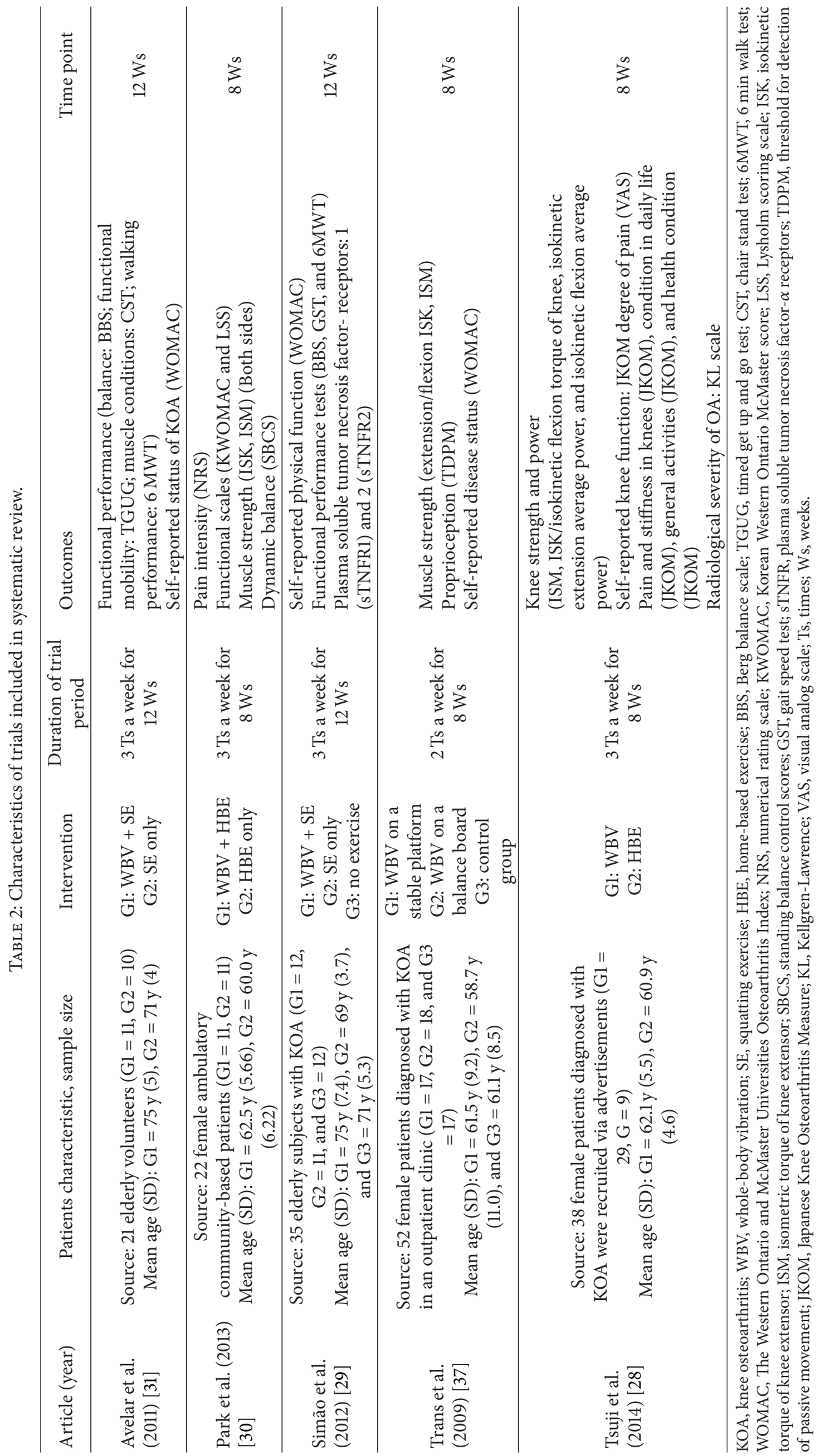


TABLE 3: The Cochrane Collaboration's tool of assessing risk of bias for methodological assessment.

\begin{tabular}{|c|c|c|c|c|c|c|}
\hline Article (year) & $\begin{array}{l}\text { Random sequence } \\
\text { generation }\end{array}$ & $\begin{array}{l}\text { Allocation } \\
\text { concealment }\end{array}$ & $\begin{array}{c}\text { Blinding of } \\
\text { participants and } \\
\text { personnel }\end{array}$ & $\begin{array}{l}\text { Blinding of } \\
\text { outcome } \\
\text { assessments }\end{array}$ & $\begin{array}{l}\text { Incomplete } \\
\text { outcome data }\end{array}$ & $\begin{array}{l}\text { Selective } \\
\text { reporting }\end{array}$ \\
\hline Avelar et al. (2011) [31] & Low & High & High & Low & High & Unclear \\
\hline Park et al. (2013) [30] & Low & High & High & Unclear & Low & Unclear \\
\hline Simão et al. (2012) [29] & Low & Low & High & Low & High & Unclear \\
\hline Trans et al. (2009) [37] & Low & Low & High & Low & High & Unclear \\
\hline Tsuji et al. (2014) [28] & Low & High & High & Low & High & Unclear \\
\hline
\end{tabular}

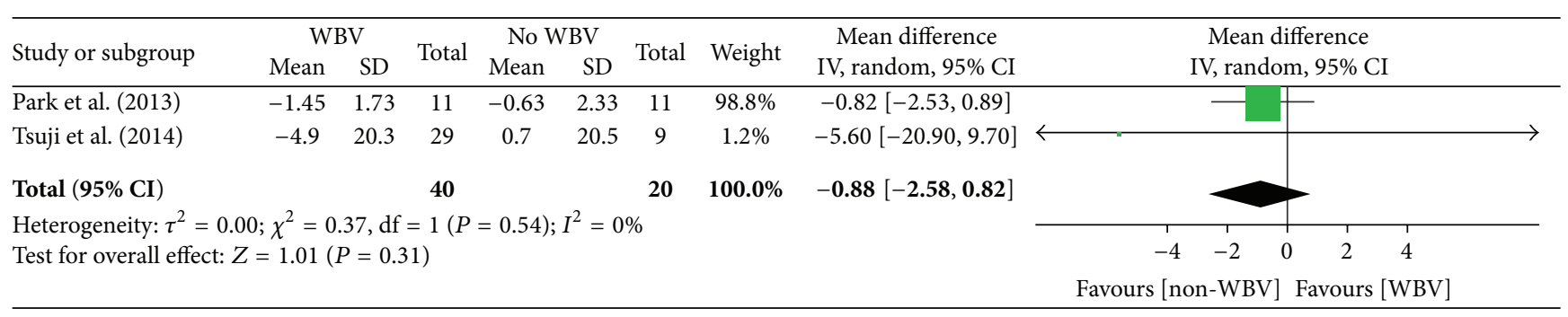

FIgURE 2: Pain intensity (evaluated by VAS or NRS) for WBV combined with other forms of exercises.

An improvement in pain intensity was observed for both trials but did not reach statistical significance in the meta-analysis $(-0.88$ points $(\mathrm{CI},-2.58$ to 0.82$), P=0.31$ ) (Figure 2). This result was consistent with the conclusion of Tsuji et al. [28]. The analysis in this study also showed that heterogeneity is low $\left(I^{2}=0 \%\right)$.

3.4.2. Functional Performance. In the five concluded studies [28-31, 37], functional performance was evaluated by various methods including BBS $[29,39], 6 \mathrm{MWT}[29,39]$, TGUG test [28, 31], $10 \mathrm{~m}$ walking time [29], KWOMAC [30], and LSS [30].

BBS was significantly improved with WBV exercise compared with other interventions without WBV (2.96 points (CI, 1.29 to 4.62 ), $P=0.0005<0.001$ ) (Figure 3(a)). One study [39] comparing WBV combined with squat exercise and squat-only exercise found negative effects for WBV combined with squat exercise on improving BBS and 6MWT. By contrast, another study [29] comparing WBV combined with squat exercise and squat-only exercise, as well as a no exercise group, reported a positive effect. This result is in accordance with the conclusions of one included trial [29].

Although we failed to find a significant difference in 6MWT (9.55 points (CI, -15.61 to 34.72$), P=0.46$ ) (Figure 3(b)), this result was in accordance with the conclusions of another trial [31]. By contrast, the heterogeneity of $6 \mathrm{MWT}$ was substantially moderate $\left(I^{2}=52 \%\right)$. This outcome might be caused by differences in the control methods between the two analyzed trials $[29,31]$. The squatonly exercise routine was used as the control treatment in one trial [31], whereas the other trial [29] had other different notreatment groups.

Two of the five included studies $[28,31]$ reported the effect of WBV according to functional ability by TGUG.
One study [31] reported a negative result in TGUG, whereas another one [28] reported that WBV had a positively significant difference in decreasing TGUG time. On the basis of these two trials, we failed to record a statistically significant difference in the meta-analysis between WBV and other exercise or placebo exercise routines in decreasing TGUG time $(-0.02$ points $(\mathrm{CI},-0.67$ to 0.63$), P=0.94)$ (Figure $3(\mathrm{c}))$.

The statistical heterogeneity for TGUG was relatively high $\left(I^{2}=82 \%\right)$. This result might be caused by the different control methods used in these two studies. Tsuji et al. [28] compared WBV with exercise at home, whereas another study [31] chose the squatting-only group as the control group in contrast to the combination of WBV and squat exercise.

3.4.3. Self-Reported Status. Among the included trials, three studies [29, 31, 37] (108 patients) demonstrated WBV and other forms of exercise by randomly assigning patients (58 and 50 patients, resp.); however, we failed to obtain the original data from one trial [37]. Therefore, the results considered self-reported status as determined by WOMAC.

A meta-analysis of three trials [29, 31, 37] showed that WBV and other forms of exercise have no significant difference in terms of WOMAC-pain ( -51.35 points (CI, -141.19 to 38.50$), P=0.26$ ) (Figure 4(a)), WOMAC-stiffness $(-4.59$ points (CI, -20.28 to 11.09$), P=0.57$ ) (Figure $4(\mathrm{~b})$ ), and WOMAC-function ( -55.05 points (CI, -150.48 to 40.37$), P=$ 0.26) (Figure 4(c)).

Substantially high levels of heterogeneity $\left(I^{2}=74 \%\right)$ in WOMAC-pain were shown in the pooled studies [29, $31,37]$ probably because of the differences among the two included studies with respect to the participant populations. The baseline level of pain, stiffness, and function observed by Simão et al. [29] was lower than those demonstrated by Avelar et al. [31]. 


\begin{tabular}{|c|c|c|c|c|c|c|c|c|c|c|}
\hline \multirow{2}{*}{ Study or subgroup } & \multicolumn{2}{|c|}{ WBV } & \multirow{2}{*}{ Total } & \multicolumn{2}{|c|}{ No WBV } & \multirow{2}{*}{ Total } & \multirow{2}{*}{ Weight } & \multirow{2}{*}{$\begin{array}{c}\text { Mean difference } \\
\text { IV, random, 95\% CI }\end{array}$} & \multirow{2}{*}{\multicolumn{2}{|c|}{$\begin{array}{c}\text { Mean difference } \\
\text { IV, random, 95\% CI }\end{array}$}} \\
\hline & Mean & $\mathrm{SD}$ & & Mean & $\mathrm{SD}$ & & & & & \\
\hline Avelar et al. (2011) & 5 & 2.76 & 10 & 2 & 1.1 & 11 & $83.1 \%$ & $3.00[1.17,4.83]$ & & - \\
\hline Simão et al. (2012) & 4.5 & 7 & 12 & 1.76 & 2.11 & 23 & $16.9 \%$ & $2.74[-1.31,6.79]$ & & \\
\hline Total $(95 \%$ CI $)$ & & & 22 & & & 34 & $100.0 \%$ & $2.96[1.29,4.62]$ & & \\
\hline \multicolumn{11}{|c|}{$\begin{array}{l}\text { Heterogeneity: } \tau^{2}=0.00 ; \chi^{2}=0.01, \mathrm{df}=1(P=0.91) ; I^{2}=0 \% \\
\text { Test for overall effect: } Z=3.47(P=0.0005)\end{array}$} \\
\hline
\end{tabular}

(a)

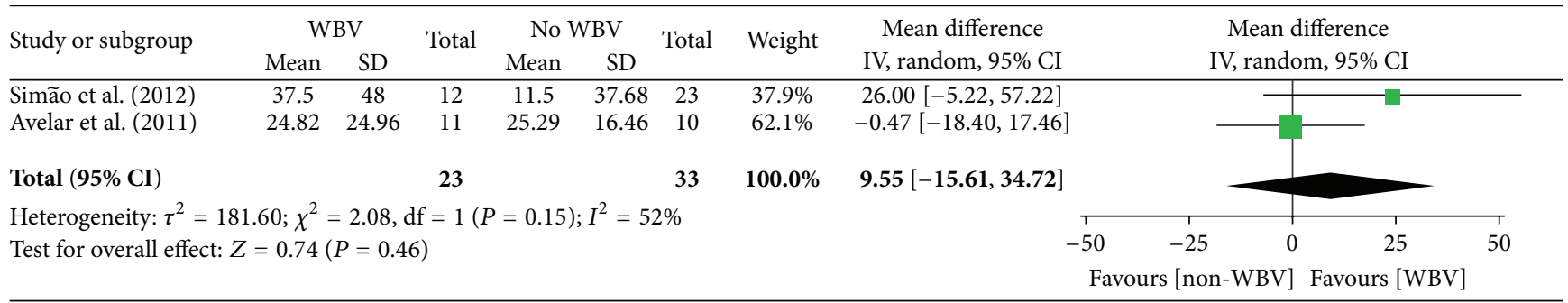

(b)

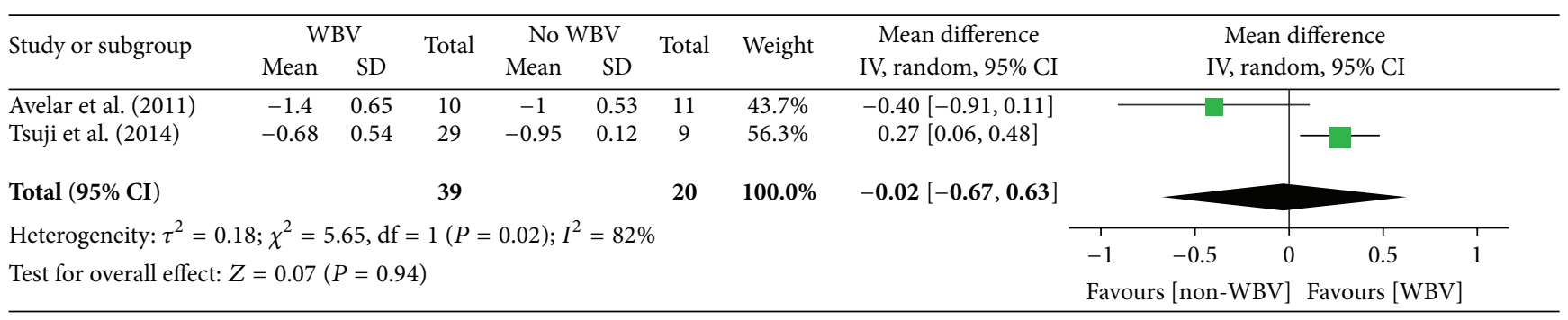

(c)

FIGURE 3: Functional performance (evaluated by BBS, 6MWT, and TGUG) for WBV compared with other forms of exercises.

3.4.4. Muscle Strength. Three RCTs [28, 30, 37] with 112 participants receiving WBV with home-based exercise and another form of exercise reported data about muscle strength, including extensor peak isokinetic torque [28, 30, 37], extensor peak isometric torque $[28,30]$, and flexor peak isokinetic torque $[28,37]$. The meta-analyses of these trials failed to reveal significant differences in extensor peak isokinetic torque (3.89 points (CI, -4.59 to 12.38$)$ ) (Figure 5(a)), extensor peak isometric torque (0.11 points (CI, -0.14 to 0.36$)$ ) (Figure $5(\mathrm{~b}))$, or flexor peak isokinetic torque $(0.07$ points $(\mathrm{CI},-0.01$ to 0.15$)$ ) (Figure 5(c)).

However, significant heterogeneity for extensor peak isokinetic torque was observed when WBV was compared with other types of exercises. The heterogeneity of the extensor peak isokinetic torque was moderate $\left(I^{2}=47 \%\right)$. Visual inspection of the forest plot revealed that three trials $[28,30,37]$ in which the control intervention was primarily performed by participants at home were the greatest possible source of heterogeneity.

3.4.5. Adverse Events. Only one RCT [39] with 28 participants receiving WBV or squat exercise reported no serious adverse effects, such as severe injuries or adverse cardiovascular effects. The evidence on adverse events of WBV is quite limited.

\section{Discussion}

Ronikeile and Costa [27] conducted a systematic review of WBV and its benefits for subjects with OA. They found that there were few publications regarding both WBV and OA, and the reported study protocols were diverse. As far as we know, this meta-analysis is the first one to evaluate the performance of WBV in KOA management.

When we applied meta-analysis to assess the consistency of evidence for the efficiency of WBV over other interventions in patients with KOA [40-42], the results were as follows:

(1) The meta-analysis showed no statistically significant differences in pain intensity evaluated by NRS and VAS $[28,30]$ between WBV combined with squat exercise and squat exercise alone.

(2) We also found no significant differences in functional performance and self-reported status. However, a statistically significant difference was found between WBV combined with squat exercise and squat exercise alone for BBS on the basis of a comparison between two trials with less than 60 patients $[29,31]$. The two trials both had high risks for incomplete outcomes, and allocation concealment was only performed in one of these two trials [29]. 


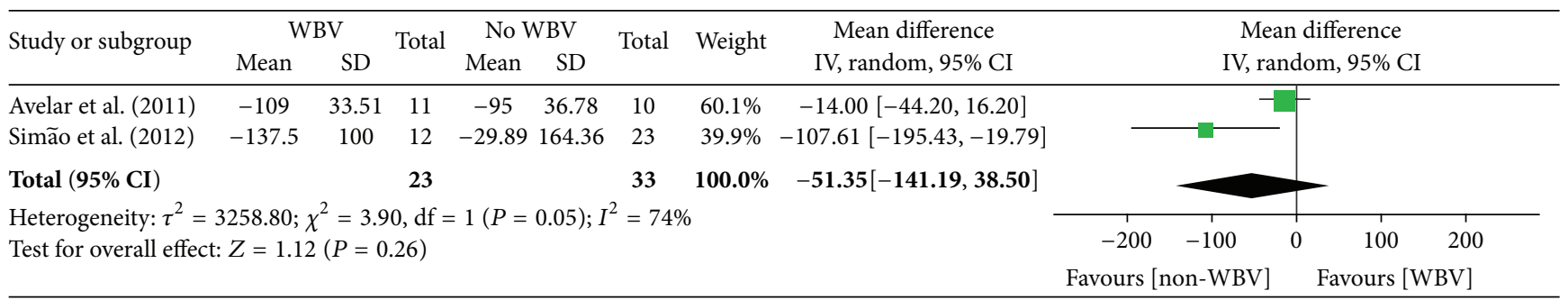

(a)

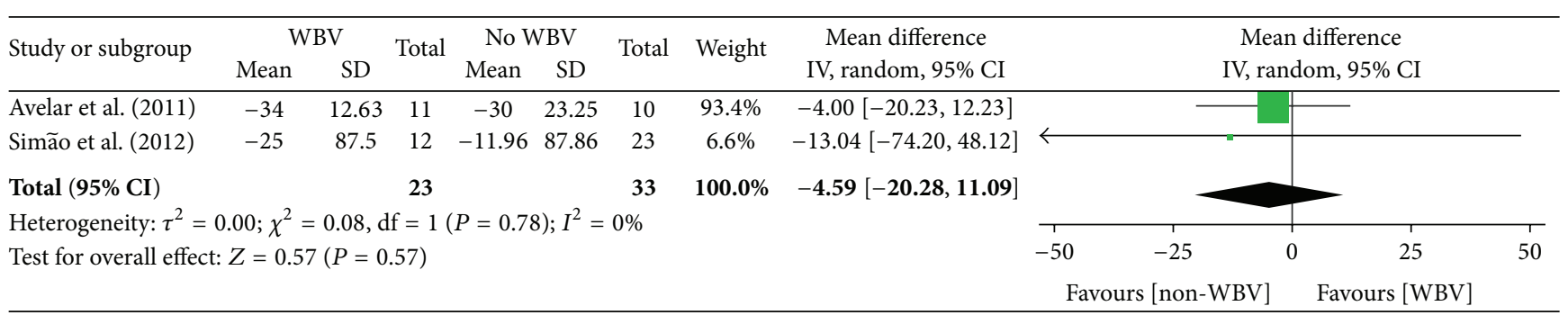

(b)

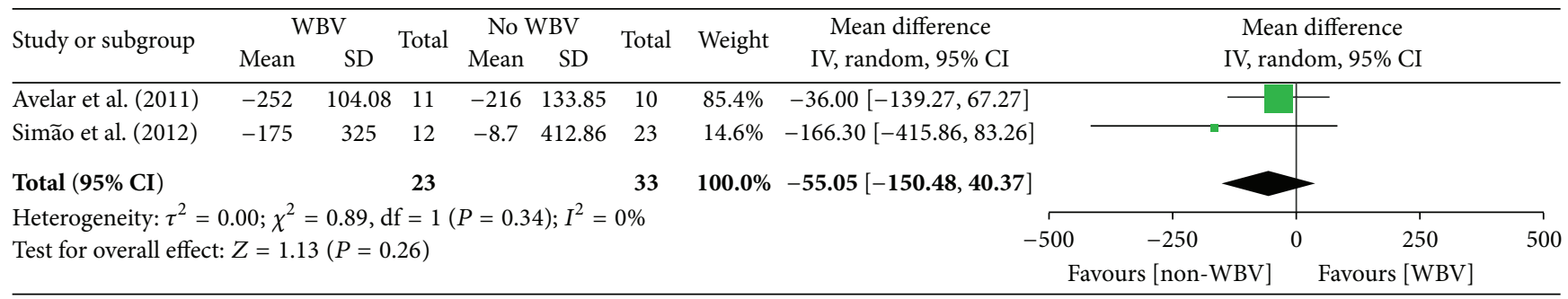

(c)

FIGURE 4: Self-reported status (evaluated by WOMAC-pain, WOMAC-stiffness, and WOMAC-function) for WBV compared with other forms of exercises.

(3) WBV is as effective as other forms of exercises in enhancing muscle strength according to an evaluation of 133 patients in four trials that had opposite results and significant differences with regard to sample size $[28,30,31,37]$.

(4) However, the absence of significant differences between WBV and other forms of exercise might be caused by the poor quality of studies providing data for some of the comparisons in the analysis.

4.1. Strengths. Our study has several strengths. We collected the data by applying a large-scale search scheme on the basis of common routines: retrieval of diversiform citation databases, recruitment of professors for published or unpublished studies, searching for document registries, and abstracting the references of the included trials. Furthermore, we did not set any limitations on year, language, status, and publication date. This strategy meant that our summary should represent the present status of all relevant research. Our review was also applied on the basis of previously published protocols and methodological schemes. The consequences of the primary outcomes of our review, including pain intensity, functional performance, and self-reported status, were reasonable. Two of the studies included in this analysis $[28,30]$ had an evaluation of pain intensity, four of the studies included in this literature $[28,29,31,37]$ had a measure of functional performance, and three trials $[29,31$, 37] reported self-reported status although only two of them provided original data for the meta-analysis of self-reported status $[29,31]$.

4.1.1. Study Limitations. First, because of the methodological weaknesses in all of the studies [28-31,37] included in our analysis, this systematic review is fundamentally limited. The risk of bias was considered high in the included studies. The most common methodological drawback in the included trials was the lack of patient and personnel blinding. However, given the characteristics of the therapeutic interventions, keeping the patients blind to the study procedures was almost impossible.

Second, we were unable to retrieve all of the original data that was needed for the analysis of the included studies [37]. The lack of some of the original data must be considered a limitation of this review because whether or not these missing results could alter the conclusion remains unknown.

Third, the population of the included trials was relatively small to the extent that none of the studies in this review enrolled 100 or more participants. These trials [28-31, 37] with a small population size were insufficient to show 


\section{Abbreviations}

$\begin{array}{ll}\text { 6MWT: } & \text { 6 min walk test } \\ \text { BBS: } & \text { Berg balance scale } \\ \text { CI: } & \text { Confidence interval } \\ \text { CST: } & \text { Chair stand test } \\ \text { GST: } & \text { Gait speed test } \\ \text { ISK: } & \text { Isokinetic torque of knee extensor } \\ \text { ISM: } & \text { Isometric torque of knee extensor } \\ \text { JKOM: } & \text { Japanese Knee Osteoarthritis Measure } \\ \text { KL: } & \text { Kellgren-Lawrence } \\ \text { KOA: } & \text { Knee osteoarthritis } \\ \text { KWOMAC: } & \text { Korean Western Ontario McMaster score } \\ \text { LSS: } & \text { Lysholm scoring scale } \\ \text { NRS: } & \text { Numerical rating scale } \\ \text { OA: } & \text { Osteoarthritis } \\ \text { RCTs: } & \text { Randomized controlled trials } \\ \text { SBCS: } & \text { Standing balance control scores } \\ \text { SD: } & \text { Standard deviation } \\ \text { SE: } & \text { Squatting exercise } \\ \text { sTNFR: } & \text { Plasma soluble tumor necrosis factor- } \alpha \\ & \text { receptors } \\ \text { TDPM: } & \text { Threshold for detection of passive } \\ \text { TUGT: } & \text { movement } \\ \text { VAS: } & \text { Timed get up and go test } \\ \text { WBV: } & \text { Whole-body vibration } \\ \text { WOMAC: } & \text { The Western Ontario and McMaster } \\ & \text { Universities Osteoarthritis Index. } \\ & \end{array}$

\section{Disclosure}

Xin $\mathrm{Li}$ is the first author, Xue-Qiang Wang is the co-first author, and Bing-Lin Chen and Ling-Yan Huang are the coauthors.

\section{Conflict of Interests}

The authors declare that there is no conflict of interests regarding the publication of this paper.

\section{Authors' Contribution}

Xin Li, Xue-Qiang Wang, and Yu Liu conceived and designed the study. Xin Li, Yu Liu, Xue-Qiang Wang, and Bing-Lin Chen collected the data. Xin Li, Xue-Qiang Wang, Yu Liu, Ling-Yan Huang, and Bing-Lin Chen interpreted the data. Xin Li drafted the paper in collaboration with Xue-Qiang Wang and Yu Liu.

\section{Acknowledgments}

The authors would like to thank Yu-shan Yue for methodological assistance during the protocol. This study was supported by Innovation Program of Shanghai Municipal Education Commission (15ZZ084), Shanghai Committee of Science and Technology (14490503800), Shanghai Youth Science and Technology Sail Project (15YF1411400), and Innovation Program of Shanghai University of Sport (yjscx2014008).

\section{References}

[1] T. Neogi, "The epidemiology and impact of pain in osteoarthritis," Osteoarthritis and Cartilage, vol. 21, no. 9, pp. 1145-1153, 2013.

[2] L. N. Reynard and J. Loughlin, "Genetics and epigenetics of osteoarthritis," Maturitas, vol. 71, no. 3, pp. 200-204, 2012.

[3] J. F. M. Holla, M. van der Leeden, M. W. Heymans et al., "Three trajectories of activity limitations in early symptomatic knee osteoarthritis: a 5-year follow-up study," Annals of the Rheumatic Diseases, vol. 73, no. 7, pp. 1369-1375, 2014.

[4] V. L. Johnson and D. J. Hunter, "The epidemiology of osteoarthritis," Best Practice \& Research Clinical Rheumatology, vol. 28, pp. 5-15, 2014.

[5] D. T. Felson, "Osteoarthritis: priorities for osteoarthritis research: much to be done," Nature Reviews Rheumatology, vol. 10, no. 8, pp. 447-448, 2014.

[6] A. A. Andrianakos, L. K. Kontelis, D. G. Karamitsos et al., "Prevalence of symptomatic knee, hand, and hip osteoarthritis in Greece. The ESORDIG study," The Journal of Rheumatology, vol. 33, no. 12, pp. 2507-2513, 2006.

[7] E. Losina, A. D. Paltiel, A. M. Weinstein et al., "Lifetime medical costs of knee osteoarthritis management in the United States: impact of extending indications for total knee arthroplasty," Arthritis Care and Research, vol. 67, no. 2, pp. 203-215, 2015.

[8] Y. Shimura, H. Kurosawa, Y. Sugawara et al., "The factors associated with pain severity in patients with knee osteoarthritis vary according to the radiographic disease severity: a crosssectional study," Osteoarthritis and Cartilage, vol. 21, no. 9, pp. 1179-1184, 2013.

[9] M. Cross, E. Smith, D. Hoy et al., "The global burden of hip and knee osteoarthritis: estimates from the global burden of disease 2010 study," Annals of the Rheumatic Diseases, vol. 73, no. 7, pp. 1323-1330, 2014.

[10] P. Piscitelli, G. Iolascon, G. Di Tanna et al., "Socioeconomic burden of total joint arthroplasty for symptomatic hip and knee osteoarthritis in the Italian population: a 5-year analysis based on hospitalization records," Arthritis Care \& Research, vol. 64, no. 9, pp. 1320-1327, 2012.

[11] M. Wortley, S. Zhang, M. Paquette et al., "Effects of resistance and Tai Ji training on mobility and symptoms in knee osteoarthritis patients," Journal of Sport and Health Science, vol. 2, no. 4, pp. 209-214, 2013.

[12] S. S. Rees, A. J. Murphy, and M. L. Watsford, "Effects of whole body vibration on postural steadiness in an older population," Journal of Science and Medicine in Sport, vol. 12, no. 4, pp. 440444, 2009.

[13] L. J. Connolly, S. Scott, M. Mohr et al., "Effects of small-volume soccer and vibration training on body composition, aerobic fitness, and muscular PCr kinetics for inactive women aged 2045," Journal of Sport and Health Science, vol. 3, no. 4, pp. 284292, 2014.

[14] E. Tankisheva, A. Bogaerts, S. Boonen, H. Feys, and S. Verschueren, "Effects of intensive whole-body vibration training on muscle strength and balance in adults with chronic stroke: a randomized controlled pilot study," Archives of Physical Medicine and Rehabilitation, vol. 95, no. 3, pp. 439-446, 2014. 
[15] P. J. Marín, C. M. Ferrero, H. Menéndez, J. Martín, and A. J. Herrero, "Effects of whole-body vibration on muscle architecture, muscle strength, and balance in stroke patients: a randomized controlled trial," The American Journal of Physical Medicine and Rehabilitation, vol. 92, no. 10, pp. 881-888, 2013.

[16] S. M. El-Shamy, "Effect of whole-body vibration on muscle strength and balance in diplegic cerebral palsy: a randomized controlled trial," American Journal of Physical Medicine \& Rehabilitation, vol. 93, no. 2, pp. 114-121, 2014.

[17] Y. Osawa, Y. Oguma, and N. Ishii, "The effects of wholebody vibration on muscle strength and power: a meta-analysis," Journal of Musculoskeletal \& Neuronal Interactions, vol. 13, no. 3, pp. 380-390, 2013.

[18] M. Y. C. Pang, R. W. K. Lau, and S. P. Yip, “The effects of wholebody vibration therapy on bone turnover, muscle strength, motor function, and spasticity in chronic stroke: a randomized controlled trial," European Journal of Physical and Rehabilitation Medicine, vol. 49, no. 4, pp. 439-450, 2013.

[19] S. von Stengel, W. Kemmler, K. Engelke, and W. A. Kalender, "Effect of whole-body vibration on neuromuscular performance and body composition for females 65 years and older: a randomized-controlled trial," Scandinavian Journal of Medicine \& Science in Sports, vol. 22, no. 1, pp. 119-127, 2012.

[20] R. Bertuzzi, L. A. Pasqua, S. Bueno et al., "Strength-training with whole-body vibration in long-distance runners: a randomized trial," International Journal of Sports Medicine, vol. 34, no. 10, pp. 917-923, 2013.

[21] T. P. Furness and W. E. Maschette, "Influence of whole body vibration platform frequency on neuromuscular performance of community-dwelling older adults," The Journal of Strength \& Conditioning Research, vol. 23, no. 5, pp. 1508-1513, 2009.

[22] W. Kemmler, S. V. Stengel, S. Mayer, M. Niedermayer, C. Hentschke, and W. A. Kalender, "Effect of whole body vibration on the neuromuscular performance of females 65 years and older. One-year results of the controlled randomized ELVIS study," Zeitschrift für Gerontologie und Geriatrie, vol. 43, no. 2, pp. 125-132, 2010.

[23] D. Perchthaler, S. Grau, and T. Hein, "Evaluation of a six-week whole-body vibration intervention on neuromuscular performance in older adults," Journal of Strength and Conditioning Research, vol. 29, no. 1, pp. 86-95, 2015.

[24] F. M. H. Lam, R. W. K. Lau, R. C. K. Chung, and M. Y. C. Pang, "The effect of whole body vibration on balance, mobility and falls in older adults: a systematic review and meta-analysis," Maturitas, vol. 72, no. 3, pp. 206-213, 2012.

[25] Osteoarthritis: National Clinical Guideline for Care and Management in Adults, Royal College of Physicians of London, London, UK, 2008.

[26] L. Zhang, C. Weng, M. Liu, Q. Wang, L. Liu, and Y. He, "Effect of whole-body vibration exercise on mobility, balance ability and general health status in frail elderly patients: a pilot randomized controlled trial," Clinical Rehabilitation, vol. 28, no. 1, pp. 59-68, 2014.

[27] P. Ronikeile and D. Costa, "Whole-body vibration and benefits for people with osteoarthritis: a system review," International Journal of Mathematics and Mathematical Sciences, vol. 6, no. 9, pp. 201-210, 2014.

[28] T. Tsuji, J. Yoon, T. Aiba, A. Kanamori, T. Okura, and K. Tanaka, "Effects of whole-body vibration exercise on muscular strength and power, functional mobility and self-reported knee function in middle-aged and older Japanese women with knee pain," The Knee, vol. 21, no. 6, pp. 1088-1095, 2014.
[29] A. P. Simão, N. C. Avelar, R. Tossige-Gomes et al., "Functional performance and inflammatory cytokines after squat exercises and whole-body vibration in elderly individuals with knee osteoarthritis," Archives of Physical Medicine and Rehabilitation, vol. 93, no. 10, pp. 1692-1700, 2012.

[30] Y. G. Park, B. S. Kwon, J.-W. Park et al., "Therapeutic effect of whole body vibration on chronic knee osteoarthritis," Annals of Rehabilitation Medicine, vol. 37, no. 4, pp. 505-515, 2013.

[31] N. C. P. Avelar, A. P. Simão, R. Tossige-Gomes et al., "The effect of adding whole-body vibration to squat training on the functional performance and self-report of disease status in elderly patients with knee osteoarthritis: a randomized, controlled clinical study," Journal of Alternative and Complementary Medicine, vol. 17, no. 12, pp. 1149-1155, 2011.

[32] J. P. T. Higgins, D. G. Altman, P. C. Gøtzsche et al., "The Cochrane Collaboration's tool for assessing risk of bias in randomised trials," British Medical Journal, vol. 343, Article ID d5928, 2011.

[33] L. Hopp, "Risk of bias reporting in Cochrane systematic reviews," International Journal of Nursing Practice, 2014.

[34] J. P. T. Higgins, S. G. Thompson, J. J. Deeks, and D. G. Altman, "Measuring inconsistency in meta-analyses," The British Medical Journal, vol. 327, no. 7414, pp. 557-560, 2003.

[35] M. J. Parkes, N. Maricar, M. Lunt et al., "Lateral wedge insoles as a conservative treatment for pain in patients with medial knee osteoarthritis: a meta-analysis," The Journal of the American Medical Association, vol. 310, no. 7, pp. 722-730, 2013.

[36] H. Balshem, M. Helfand, H. J. Schünemann et al., "GRADE guidelines: 3 . Rating the quality of evidence," Journal of Clinical Epidemiology, vol. 64, no. 4, pp. 401-406, 2011.

[37] T. Trans, J. Aaboe, M. Henriksen, R. Christensen, H. Bliddal, and H. Lund, "Effect of whole body vibration exercise on muscle strength and proprioception in females with knee osteoarthritis," Knee, vol. 16, no. 4, pp. 256-261, 2009.

[38] C. Larroy, "Comparing visual-analog and numeric scales for assessing menstrual pain," Behavioral Medicine, vol. 27, no. 4, pp. 179-181, 2002.

[39] T. Osugi, J. Iwamoto, M. Yamazaki, and M. Takakuwa, "Effect of a combination of whole body vibration exercise and squat training on body balance, muscle power, and walking ability in the elderly," Therapeutics and Clinical Risk Management, vol. 10, no. 1, pp. 131-138, 2014.

[40] R. Tanaka, J. Ozawa, N. Kito, and H. Moriyama, "Efficacy of strengthening or aerobic exercise on pain relief in people with knee osteoarthritis: a systematic review and meta-analysis of randomized controlled trials," Clinical Rehabilitation, vol. 27, no. 12, pp. 1059-1071, 2013.

[41] R. Tanaka, J. Ozawa, N. Kito, and H. Moriyama, "Effect of the frequency and duration of land-based therapeutic exercise on pain relief for people with knee osteoarthritis: a systematic review and meta-analysis of randomized controlled trials," Journal of Physical Therapy Science, vol. 26, no. 7, pp. 969-975, 2014.

[42] J. Ye, S. Cai, W. Zhong, S. Cai, and Q. Zheng, "Effects of tai chi for patients with knee osteoarthritis: a systematic review," Journal of Physical Therapy Science, vol. 26, no. 7, pp. 1133-1137, 2014. 


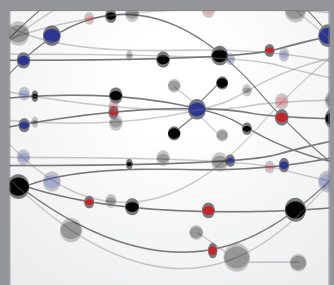

The Scientific World Journal
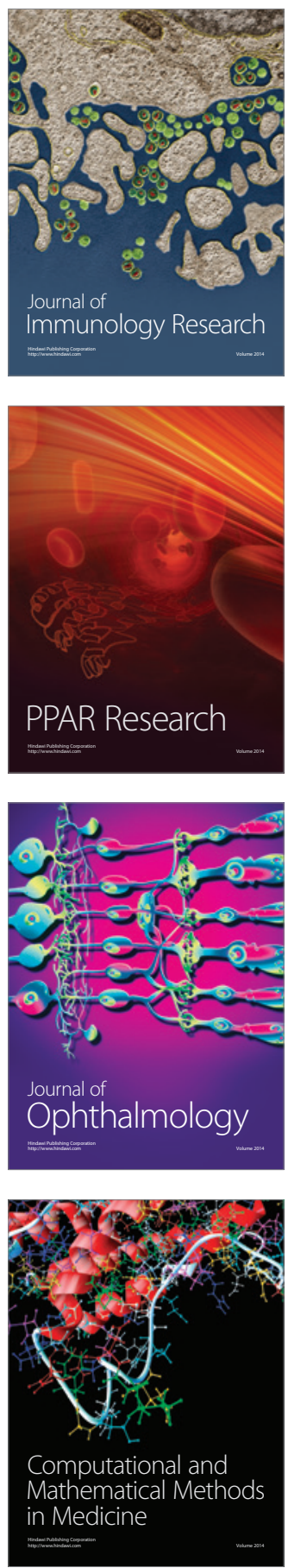

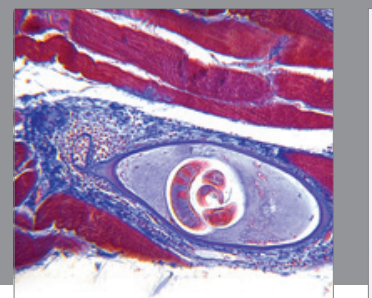

Gastroenterology

Research and Practice
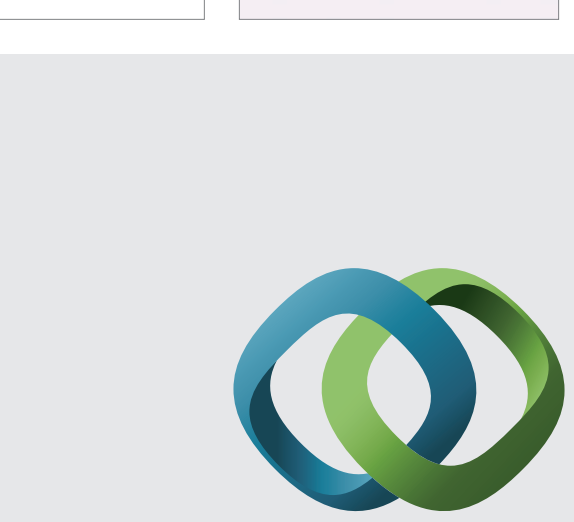

\section{Hindawi}

Submit your manuscripts at

http://www.hindawi.com
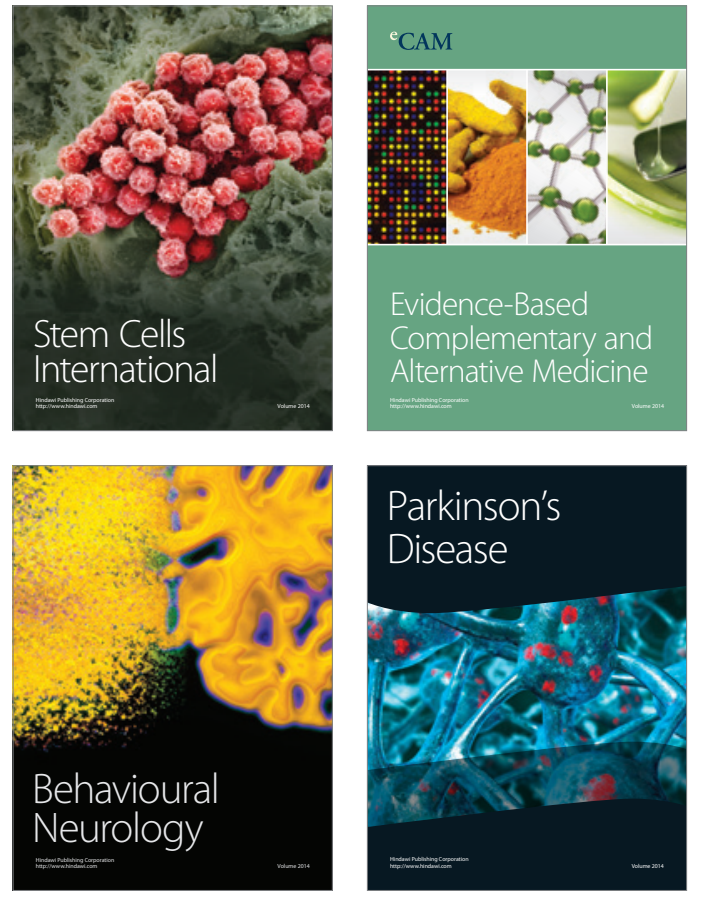
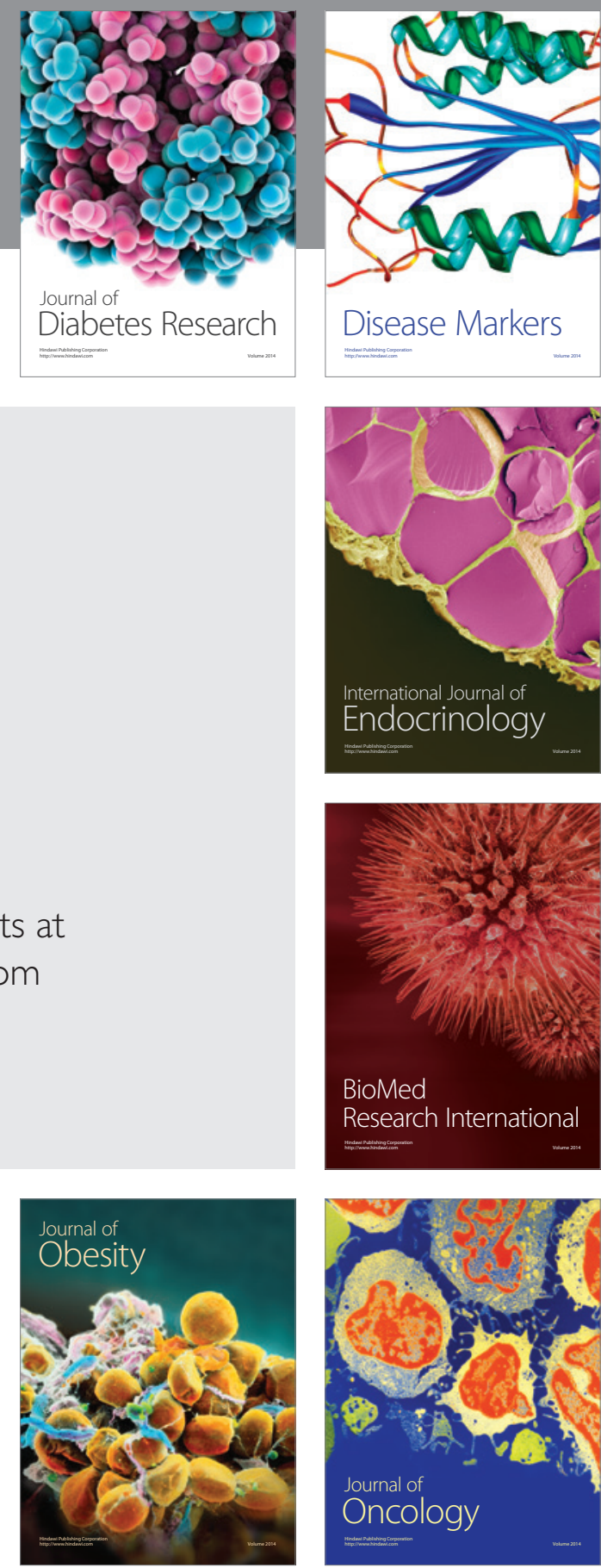

Disease Markers
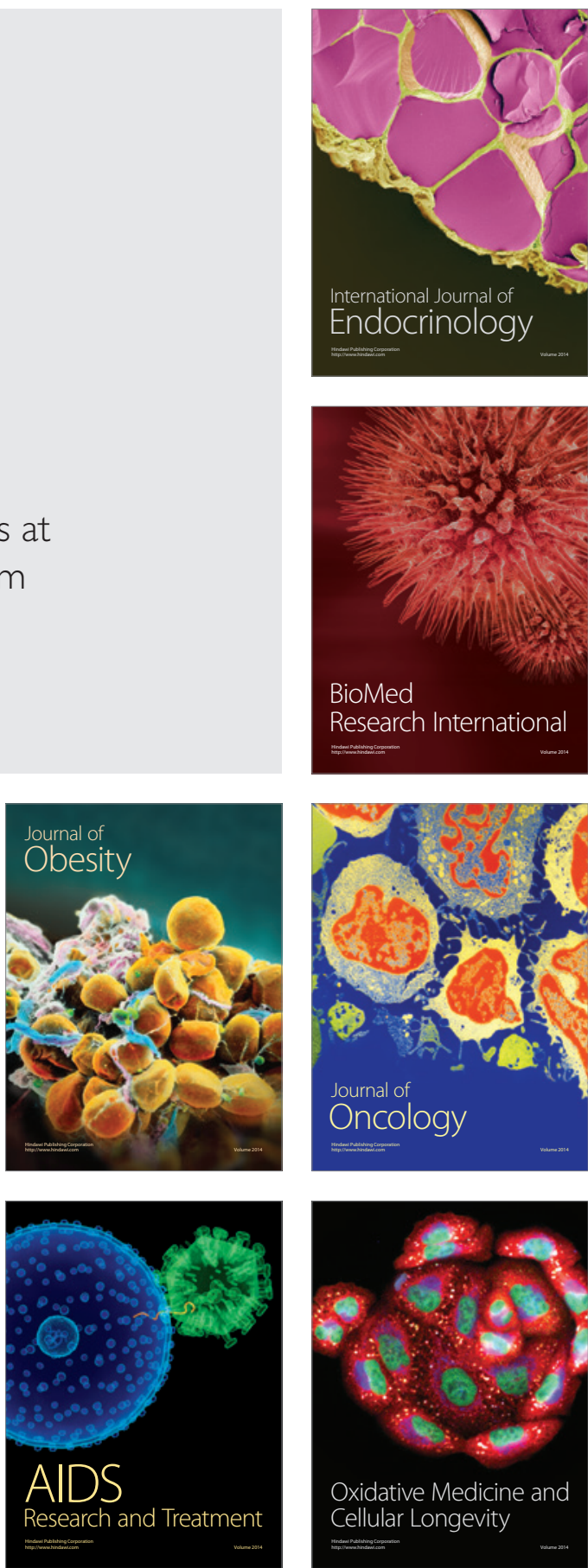